\title{
SIMULATED CHAOS IN BULLWHIP EFFECT
}

\section{DOI: 10.17261/Pressacademia.2015111603}

\section{Tunay Aslan ${ }^{1}$}

1Sakarya University, Turkey. tunay_aslan@hotmail.com

\section{Keywords}

Supply chain, Bullwhip Effect, Chaos Theory, Lyapunov Exponent, Time Series, poisson number generation.

\begin{abstract}
The main purpose of the research is to investigate nonlinear dynamics in bullwhip effect and search chaotic behavior. In the paper, a generalized supply chain model is simulated with safety stock regulations to expose the bullwhip effect. A seasonal demand model which fits Poisson distribution is utilized to generate orders from customers to retailers, continuously to distributors and a single factory. Using largest Lyapunov exponent analysis, orders are reconstructed in phase space and investigated chaotic behavior variations. Although it is assumed that increasing fluctuations of demand cause chaos and unpredictability, it is seen that predictability increases in bullwhip effect. In chaotic research aspect, demands from customers are still more chaotic than orders reach to the factory. Due to data generation, it is still a realization of a supply chain, therefore working on real data is suggested. The paper includes implications for giving ideas of nonlinear dynamics of bullwhip effect. This paper provides a novel approach to supply chains with comparing dynamics of demands and orders to identify which exhibits more chaotic behavior.
\end{abstract}

\section{INTRODUCTION}

Supply Chain is a complex system in which a nonlinear time series model exists, known as Bullwhip Effect. Bullwhip Effect is a problematic situation in a supply chain which is clarified as increasing variability of demands as a result of keeping safety stocks (Forrester, 1961). The results of bullwhip effect are summarized as; excess inventories, surplus or short capacities, higher and unnecessary inventory costs. Moving up the supply chain from customers to factory, orders are increased and demonstrate more fluctuations. These oscillations and amplifications are expected to reduce predictability of the orders moving upstream in the chain. Therefore, it is assumed that high fluctuation causes ambiguity which is chaotic and less predictable.

Unpredictability of a dynamic system is calculated with Lyapunov exponent analysis and positive largest Lyapunov exponent indicates chaos (Rosenstein et al., 1993). Although there is no constant definition of chaos, it can be simplified as aperiodic, long-term behavior of a dynamic system that exhibits sensitive dependence on initial conditions (Sprott, 2003).

There are a few number of studies have researched chaotic dynamics of bullwhip effect. Two very similar papers propose to adapt bullwhip effect to Lorenz equations (Renzhong and Gongyun, 2011; Lei et al., 2006). They expressed the mathematical model of bullwhip effect from end customers to producers in three difference equations and transformed to 
Lorenz model. Anne et al. (2009) also researched chaos in supply chain using Lorenz model.

$\mathrm{Ma}$ and Feng (2008) presented mathematical form of bullwhip effect using demand and prices, constructed time delay vectors and investigated bifurcations. Makui and Madadi (2007) used largest Lyapunov exponent analysis to investigate chaos in bullwhip effect however; the numerical example in their paper is too short for Lyapunov exponent convergence. Stapleton et al (2006) published a research note and introduced chaos theory as a possible enhancement of effectiveness of a supply chain.

In this study, a supply chain model is constructed with 100 retailers, 10 distributors and one factory. We generated daily customer demand data for ten years, which fits Poisson distribution, added sinusoidal seasonality for weekdays. After data generation, we simulated orders and investigated chaotic dynamics using Lyapunov exponent method.

\section{DATA GENERATION}

The first step of our research involves constructing a simple supply chain model. Our study is based on the four level supply chain illustrated in Fig.1 whereas some articles about bullwhip effect don't take customer as a level, and place wholesaler between retailer and distributor (Machuca and Barajas, 2004).

Fig.1. Diagram of Our Supply Chain Model

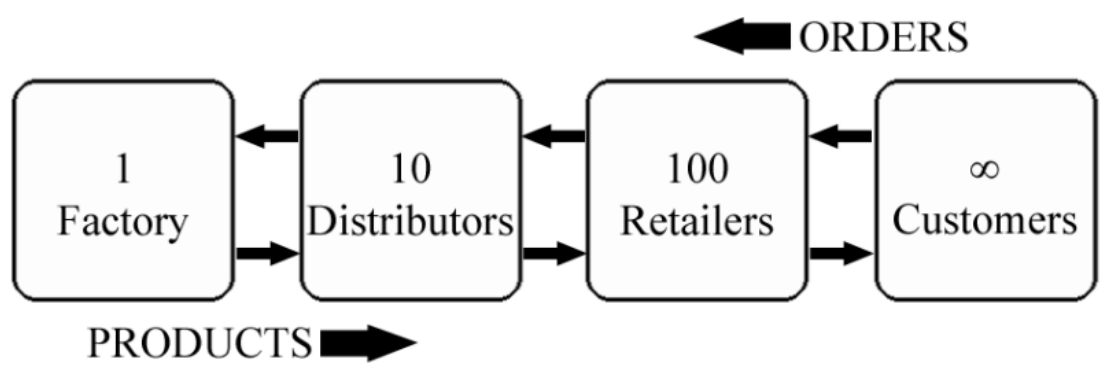

After constructing the chain system, we generated random numbers as customers' orders to retailers, which fit homogenous Poisson distribution. The Poisson distribution expresses the independent probability of an event occurring in an interval of time, with certain average (Gullberg, 1997). This distribution can be used conveniently while generating arrivals or orders.

Poisson distribution focuses on a random variable that is discrete and countable. The expected value of an event's occurrence, which is independent of the previous event, is shown as $\alpha$. The probability of a positive integer of $k$ event occurrence is given by;

$$
f(k, \alpha)=\frac{\alpha^{k} e^{-\alpha}}{k !}
$$

where $e$ is the base of the natural logarithm.

While generating $3650 \times 100$ demand data for 100 retailers, we choose $\alpha=4$ for our simulation hence mean $\mu$ and variance $\sigma^{2}$ equal to 4 . For the purpose of making data more realistic and differentiating weekdays, we added sinusoidal seasonality $S$ to the demand 
data $A$, therefore, weekends would have more demand. The sinusoidal equation is shown below:

$$
S=\left[2\left(\sin \left(\frac{2 \pi}{7} \bmod (A, 7)\right)+1\right)\right]
$$

After taking the integer value of the result, no subtraction would take place; only 0 to 3 demands would be added to the daily demand data. After generation of the data, we started our simulation to expose bullwhip effect.

\section{SIMULATION}

The simulation model has these assumptions; all retailers have 10 safety stock capacity and order to related distributor when stock levels drop under 5, all distributors have 100 capacity and order to factory when levels are below 50. As expected, order oscillations and values are increased while moving up to factory, illustrated in Fig. 2.

Fig.2. Simulated Bullwhip Effect

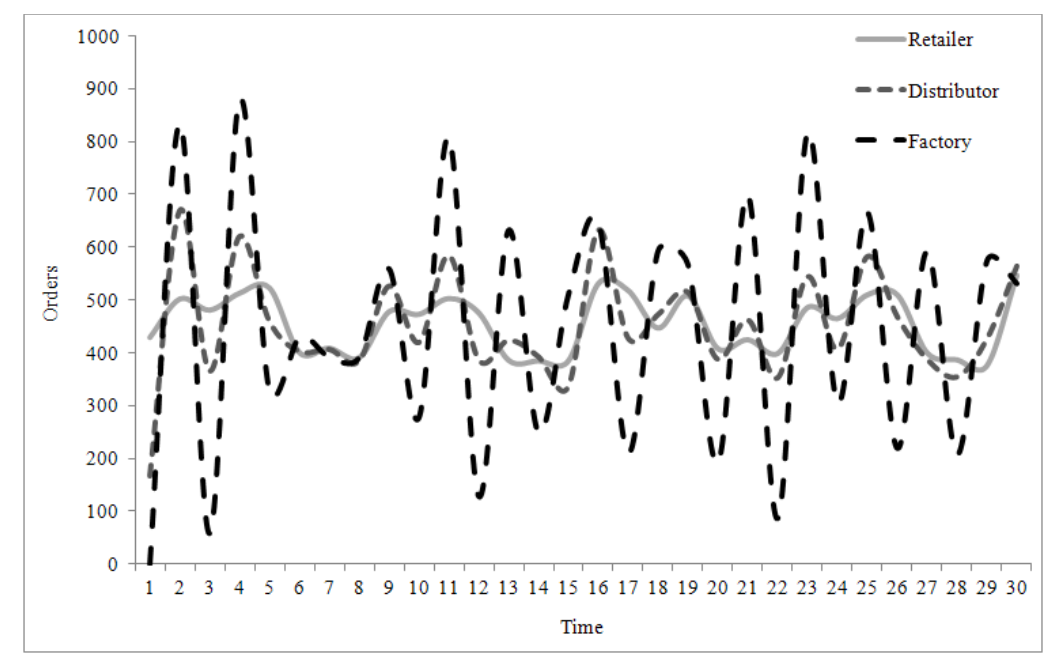

These values are daily consolidated data of demands from the customers (retailer curve), of orders to distributor (distributor curve) and to factory (factory curve). As seen on the figure, fluctuations increase severely, that should make the system become more chaotic and unpredictable. However, without Lyapunov exponent analysis, it would be a misjudgment.

Additionally, in order to confirm the output of the simulation, we run the system 10 times starting from data generation and compared largest Lyapunov exponents with student ttest. 


\section{LYAPUNOV EXPONENT ANALYSIS}

Calculation of the largest Lyapunov exponent is mostly used method to discover chaotic behavior where sign of the largest positive exponent indicates chaos and value of the positive exponent demonstrate how chaotic the system is.

Although there are numerous major studies about calculating largest Lyapunov exponent from time series (Wolf et. al., 1985; Echmann et. al., 1986; Sato et. al, 1987; Brown et. al, 1991; Abarbanel et. al., 1993), each method offers only an estimation of the Lyapunov exponent, not the exact value.

However, estimations are useful when comparing the largest Lyapunov exponents of multiple data sets. The standardized Lyapunov analysis begins with phase space reconstruction (Kantz and Schreiber, 1997).

$$
X(t)=\{x(t), x(t+\tau) . . x[t+(m-1) \tau]\}
$$

In this equation $m$ is embedding dimension and $\tau$ is embedding delay. Selecting these parameters correctly is the main issue for single time series. The embedding delay is estimated using autocorrelation and mutual information functions. Autocorrelation function displays linear dependency between $X(t)$ and $X(t+\tau)$. The first zero-crossing point of autocorrelation graph gives proper delay value (Buzug and Pfister, 1992) and also the first minimum of mutual information function usually should point the same value (Fraser ve Swimney, 1986).

The embedding dimension is usually estimated using false nearest neighbors in accordance with Takens' theorem (Takens, 1981). This dimension identifies the factors that affect main variable of the time series, additionally determines the Lyapunov spectrum. In other words an $m$ dimensional system has $m$ Lyapunov exponents. The nearest neighbor of each point is found by searching for the point which minimizes the distance to a particular point. After iterations the neighbors are moving apart from each other that clarifies it was a false neighbor. At some point, false neighbors become zero; herewith the first minimum point of false nearest neighbor graph states the embedding dimension. Thus, the largest Lyapunov exponent is estimated with calculating separation rate of the neighbors (Rosenstein et. al., 1993)

\section{RESULTS}

This study depends on comparisons of Lyapunov exponents; therefore we estimated embedding dimension $m$ and embedding delay $\tau$ for the first simulation and hold them constant for the others. Calculation of $m$ and $\tau$ is illustrated in Fig.3. 
Fig.3. Example of embedding delay (on the left) and embedding dimension (on the right) graphs
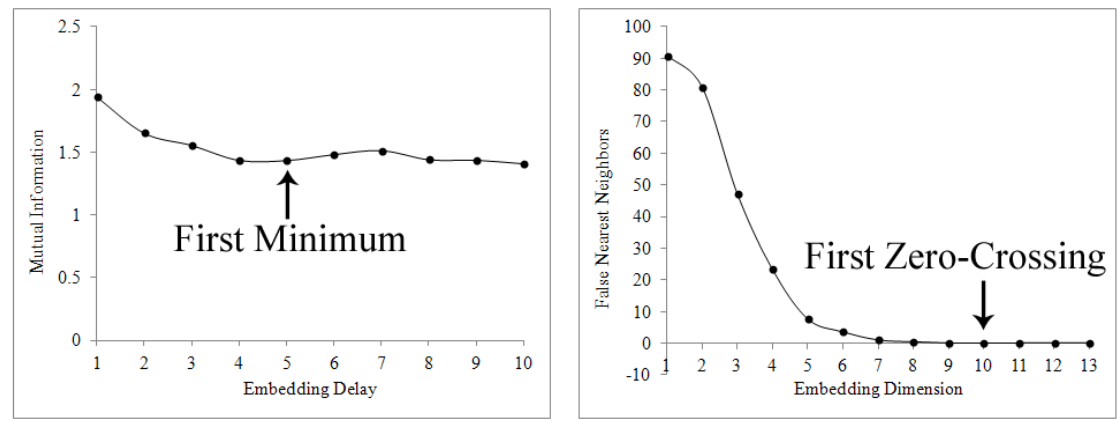

Actually, these parameters do not fluctuate over simulations, taking $m=5$ and $\tau=10$ as constants for all analysis doesn't cause inaccuracy. For the first simulation, positive Lyapunov exponents are calculated and are shown in Fig.4. There are two considerable advices to calculate largest Lyapunov exponent from entire positive exponents: First method is numerical calculation of largest Lyapunov exponent (Schuster, 2004) which is;

$$
L L E=\lim _{n \rightarrow \infty} \frac{1}{n} \sum_{i=1}^{n} \lambda_{i}
$$

This method is useful for very large number of data set therefore we used the second method that is estimating largest Lyapunov exponents where the convergence occur (Peters, 1996). An example of approximate convergence is shown in Fig. 4. below.

Fig.4. Convergence of Largest Lyapunov Exponents

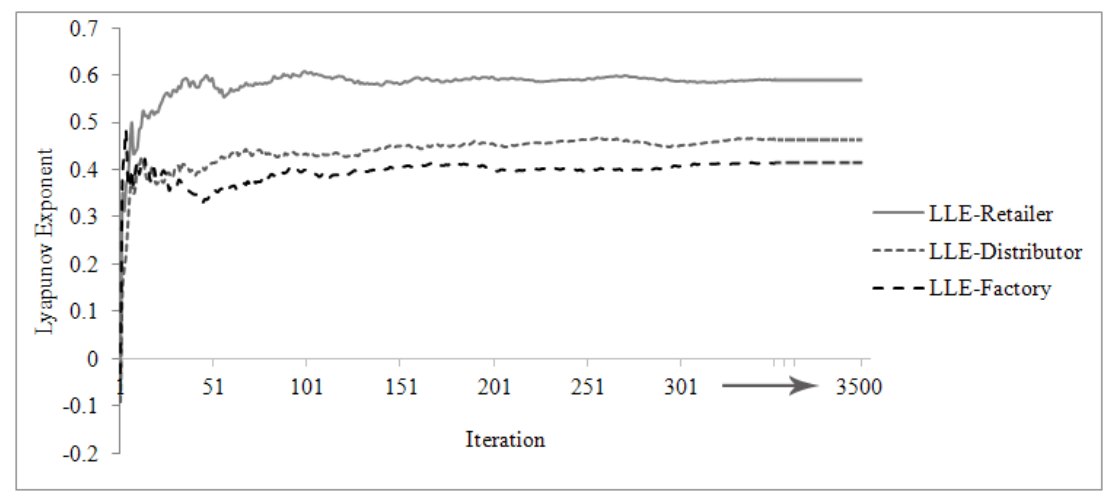

In this simulation, LLE-Retailer $\approx 0.584$, LLE-Distributor $\approx 0.466$ and LLE-Factory $\approx 0.406$ are calculated. Wherefore largest Lyapunov Exponent is an average of unpredictability or global measure of sensitivity of the system to slight changes (Williams, 1997), predictability increases unexpectedly moving up in the supply chain. The orders of factory has smaller Lyapunov exponent than others, although it has more oscillation and amplification. 
After 10 simulation sets, we achieved average largest Lyapunov exponents. The mean of these exponents is illustrated in Fig.5. with maximum and minimum levels.

Fig.5. Calculated mean, max and min values of Largest Lyapunov Exponents

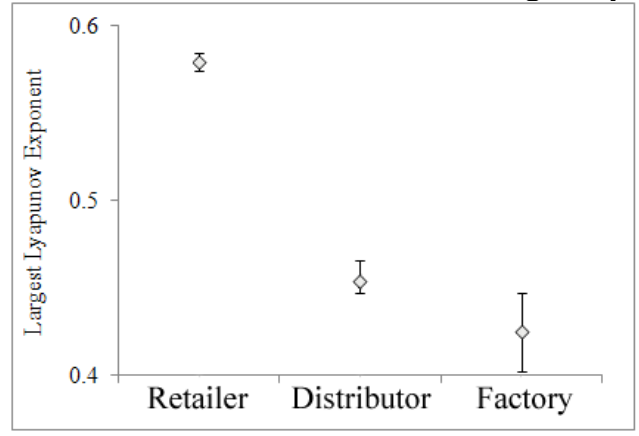

The last issue is to determine whether the differences are significant. We made student $t$ test for paired means and compared t-stats with two tailed t-critical values for 0.01 significance level. For first two means, which are retailer-distributor and retailer-factory pairs, t-stats 53.80933 and 28.50217 are significantly larger than t-critical value 3.249834. Also for distributor-factory pairs, we found t-stat 5.844 that is larger than 3.249834 likewise. The statistical results prove that Largest Lyapunov exponents and chaotic behavior decreases significantly from retailers to factory.

\section{CONCLUSION AND DISCUSSION}

Firstly, we have presented a supply chain model and seasonal Poisson data generation in this paper. As results of the simulations we reached bullwhip effect and searched whether the bullwhip exhibit chaotic behavior. Hence largest Lyapunov exponent is numerical indicator of chaos presence, by the method of phase space reconstruction, after calculating the embedding dimension and delay; we analyzed the largest Lyapunov exponents of supply chain orders.

Simulation results prove that chaotic behavior of the system decreases while moving on supply chain from retailers to factory. Largest Lyapunov exponents differ significantly after 3650 days of iterations and 10 repetitions. Generated seasonal retailer's demand data has more unpredictability and chaotic behavior while it has less fluctuation. Against all expectations, orders to distributors and to factory lead the system to behave less chaotic while more frequent and amplified oscillations are taking place.

\section{REFERENCES}

Abarbanel, H. D. I., Brown R., Sidorowich J. J., Tsimrin L. S., 1993. The Analysis of Observed Chaotic Data in Physical Systems. Reviews of Modern Physics 65(4). 1331-1392.

Anne, K. R., Chedjou, J. C., Kyamakya, K., 2009. Bifurcation analysis and synchronisation issues in a three-echelon supply chain. International Journal of Logistics Research and Applications: A Leading Journal of Supply Chain Management 12(5).

Brown R., Bryant P., Abarbanel H. D. I., 1991. Computing the Lyapunov spectrum of a dynamical system from observed time series. Phys. Rev. A 43. 2787-2806. 
Buzug, T., Pfister G., 1992. Optimal delay time and embedding dimension for delay-time coordinates by analysis of the global static and local dynamical behavior of strange attractors. Physical Review A 45(10). 7073-7084.

Eckmann, J. P., Kamphorst, S. O., Ruelle, D., Ciliberto, S., 1986. Liapunov exponents from time series. Phys. Rev. A 34(6), 4971-4979.

Forrester, J. W., 1961. Industrial Dynamics, The MIT Press, John Wiley \& Sons, New York, USA

Fraser, A. M., Swinney, H. L., 1986. Independent coordinates for strange attractors from mutual information. Physical Review A 33(2). 1134-1140.

Gullberg, J., 1997. Mathematics from the birth of numbers. W. W. Norton, New York, U.S.A.

Kantz, H., Schreiber, T., 1997, Nonlinear Time Series Analysis. Cambridge University Press.

Lei, Z., Yi-jun, L., Yao-qun, X., 2006. Chaos Synchronization of Bullwhip Effect in a Supply Chain Conference on Management Science and Engineering, ICMSE '06. 2006 International. 557 - 560.

Ma, J., Feng, Y., 2008. The Study of the Chaotic Behavior in Retailer's Demand Model, Discrete Dynamics in Nature and Society 2008. 1 - 12.

Machuca, J. A. D., Barajas, R. P., 2004. The impact of electronic data interchange on reducing bullwhip effect and supply chain inventory costs. Transportation Research Part E 40. 209-228

Peters, E. E., 1996. Chaos and order in the capital markets: A new view of cycles, prices, and market volatility 2 nd ed. John Wiley \& Sons Inc, New York, USA.

Renzhong, L., Gongyun, G., 2011. Research on Chaos Synchronization of Bullwhip Effect in Supply Chain, Conference on Product Innovation Management (ICPIM), 2011 6th International. 398-401.

Rosenstein, M. T., Collins J. J., De Luca C. J., 1993. A practical method for calculating largest Lyapunov exponents from small data sets. Physica D 65, 117-134.

Sato, S., Sano, M., Sawada, Y., 1987. Practical methods of measuring the generalized dimension and the largest Lyapunov exponent in high dimensional chaotic systems, Progress of Theoretical Physics. 77(1). 1-5.

Schuster, H. G., Just, W., 2004. Deterministic Chaos, An Introduction. Wiley VCH.

Sprott, J. C., 2003. Chaos and Time Series Analysis. Oxford University Press.

Stapleton, D., Hanna, J. B. Ross, J. R., (2006) "Enhancing supply chain solutions with the application of chaos theory", Supply Chain Management: An International Journal, Vol. 11 Iss: 2, pp.108-114

Takens, F., (1981). Detecting Strange Attractors in Turbulence. Lecture Notes in Mathematics 898. 366-381.

Williams, G. P., (1997). Chaos Theory Tamed. Joseph Henry Press. Washington D.C. U.S.A.

Wolf, A., Swift, J. B., Swinney H. L., Vastano, J. A., (1985). Determining Lyapunov exponents from a time series. Physica D 16. 285-317. 